\title{
Involving patients in enrolment decisions for acute myocardial infarction trials
}

The provenance and peer review statement in this Analysis article (BMJ 2015;351:h3791, doi:10.1136/bmj.h3791) is incorrect. The statement should have read "Not commissioned; externally peer reviewed," rather than "Commissioned; not externally peer reviewed," 